\title{
Was bringen genetische Untersuchungen für die Psychotherapie?
}

\section{Ein Interview mit Jürgen Deckert}

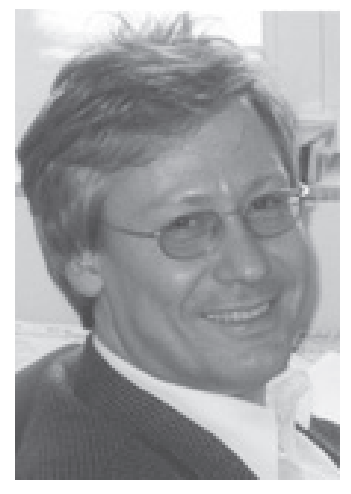

\begin{abstract}
Vor einigen Jahren hat die Entschlüsselung des menschlichen Genoms eine große Euphorie ausgelöst. Endlich könne man das komplette Erbmaterial wie ein Buch lesen. Die Ursachen von psychischen und somatischen Erkrankungen könnten so gefunden, vielleicht sogar durch Gentherapie geheilt werden - so war zumindest in der Laienpresse zu lesen. Haben sich die hohen Erwartungen, die an die Genetik gestellt wurden, erfüllt? Oder determinieren weniger Erbanlagen als vielmehr Umweltfaktoren entscheidend persönliche Entwicklung und Krankheit? Was hat die genetische Forschung für den Psychotherapeuten bisher gebracht? Zu diesen Fragen wurde einer der führenden Experten in Deutschland, Prof. Dr. Jürgen Deckert, interviewt. Er ist Direktor der Klinik und Poliklinik für Psychiatrie, Psychosomatik und Psychotherapie an der Universität Würzburg. Das Gespräch führte Prof. Dr. Tilo Kircher aus Marburg.
\end{abstract}

Wie erklärt man sich heutzutage den Zusammenhang zwischen Genen und psychischen Störungen?

Nach heutigem Kenntnisstand geht man davon aus, dass genetische Variationen eigentlich an der Entstehung aller psychischen Erkrankungen - allerdings unterschiedlich - beteiligt sind.

Wichtig dabei ist, dass der Beitrag jeder einzelnen genetischen Variation im Rahmen der komplexen Entstehung psychischer Erkrankungen gering ist. Im Gegensatz zu kausalen Genen wie sie bei mendelischen Erkrankungen wie z.B. der Chorea Huntington eine entscheidende Rolle spielen, spricht man von Suszeptibilitätsgenen.

Spielen soziale und Umweltfaktoren heute kaum mehr eine Rolle bei der Genese?

Psychosoziale und Umweltfaktoren sind bei der Entstehung psychischer Erkrankungen genauso wichtig wie genetische Variationen, allerdings ebenfalls je nach Erkrankung unterschiedlich. Während z.B. bei der Bipolaren Affektiven Erkrankung genetische Faktoren eine größere Rolle spielen, spielen bei der Entstehung der Unipolaren Depression Um- weltfaktoren eine größere Rolle. Man geht heute davon aus, dass genetische und Umweltfaktoren in der Entstehung vermittelt durch epigenetische Prozesse, z.B. Methylierung von Genen, zusammenwirken. Solche epigenetische Prozesse führen zwar nicht zu einer Sequenzveränderung der Gene, jedoch zu einer Veränderung der Umwandlung in Proteine und haben damit erhebliche funktionelle Auswirkungen. Sie können z.B. die Auswirkungen günstiger oder ungünstiger genetischer Varianten ausgleichen oder sogar in das Gegenteil umwandeln.

Aus der Presse gewinnt man den Eindruck, dass wenn erst einmal das Genom entschlüsselt ist, wir über das individuelle Risiko somatischer und psychischer Erkrankungen jeder Einzelperson Vorhersagen machen können. Was ist hier aus Sicht der Wissenschaft zu sagen?

Dies trifft nur für die sehr seltenen mendelschen Erkrankungen wie z.B. die Chorea Huntington zu. Bei den meisten psychischen wie somatischen Erkrankungen mit ihrer komplexen Entstehung ginge dies theoretisch nur bei Kenntnis aller genetischen und Umweltfaktoren und deren Zusammenwirken durch Erstellung von individuellen Risikoprofilen.

\section{KARGER}

Fax +497614520714

Information@Karger.com

www.karger.com (c) 2014 S. Karger GmbH, Freiburg

Accessible online at:

www.karger.com/ver 
Dies ist aber wegen der hohen Zahl von möglichen Faktoren und Kombinationen auf absehbare Zeit, wenn überhaupt jemals, nicht zu erwarten.

Was ist bekannt über genetische Varianten und Psychotherapie?

Hier gibt es noch sehr wenige Untersuchungen. Vorhandene Studien fokussieren in Post-hoc-Analysen auf die Rolle genetischer Variationen wie z.B. den Val/Met-Polymorphismus im COMT-Gen für zentrale psychotherapeutische Prozesse wie das Extinktionslernen. Eine Handvoll Untersuchungen, wie z.B. die des BMBF-Paniknetzes (www.paniknetz.de), hat ebenfalls in Post-hoc-Analysen den Effekt genetischer Variationen wie des Promoters VNTR (variable number tandem repeat) im MAO-A-Gen auf den Therapieeffekt untersucht.

Ist es möglich, aufgrund des Genotyps Prognosen zum individuellen Ansprechen auf Psychotherapie oder bestimmte Therapiearten wie die Kognitive Verhaltenstherapie oder tiefenpsychologische Psychotherapie abzugeben?

Die Untersuchung unseres BMBF-Paniknetzes hat gezeigt, dass Panikpatienten mit der längeren, aktiveren Variante des Promoters VNTR im MAO-A-Gen schlechter auf eine Kognitive Verhaltenstherapie ansprechen - ebenso wie in einer anderen Untersuchung Patienten mit einer Depression und der längeren, aktiveren Variante schlechter auf eine pharmakologische antidepressive Therapie ansprechen. Beide Befunde sind bisher jedoch noch nicht repliziert. Befunde zum differenziellen Ansprechen unterschiedlicher Psychotherapieverfahren gibt es bisher nicht; nicht zuletzt auch deswegen, weil es kaum Untersuchungen gibt, die unterschiedliche Psychotherapieverfahren miteinander vergleichen.
Wird man in Zukunft vor Beginn einer Psychotherapie in der Praxis erst einmal den Genotyp untersuchen - Stichwort «personalised medicine»?

Die bisherigen Befunde beruhen auf Post-hoc-Analysen. A-priori-Untersuchungen zur Beantwortung dieser Frage fehlen bisher. Vorstellbar und letztlich Ziel der Forschung auf diesem Gebiet ist es, dass in fernerer Zukunft in Ergänzung der biografischen Anamnese mit Erfassung von Life-Events, kognitiven Mustern, Beziehungsverhalten und Coping-Strategien auch genetische Varianten im Rahmen einer personalisierten Medizin zur Entscheidung für die Individualisierung von Therapien beitragen können. Hier besteht aber noch erheblicher Forschungsbedarf.

Professor Deckert, ich danke Ihnen sehr für dieses Gespräch! 